\title{
Persistent Disparities in Obesity Risk Among Public Schoolchildren from \\ Childhood Through Adolescence
}

Danhong Chen ${ }^{1, *}$, Ph.D.; Michael R. Thomsen ${ }^{1,2}$, Ph.D.; Rodolfo M. Nayga, Jr. ${ }^{1,2,3,4}$, Ph.D.; Judy L. Bennett ${ }^{5}$, M.S.

${ }^{1}$ Department of Agricultural Economics and Agribusiness, University of Arkansas, Fayetteville, AR, USA

${ }^{2}$ National Bureau of Economic Research

${ }^{3}$ Norwegian Institute of Bioeconomy Research, Norway

${ }^{4}$ Korea University, Seoul, South Korea

${ }^{5}$ Arkansas Center for Health Improvement, Little Rock, AR, USA

*Corresponding author: Danhong Chen; Address: Department of Agricultural Economics and Agribusiness, University of Arkansas, 207 Agriculture Annex Building, Fayetteville, AR 72701; Phone: 479-575-2256; Email: danhong_chen@hotmail.com 


\begin{abstract}
Introduction: Arkansas is among the poorest states and has high rates of childhood obesity. In 2003, it became the first state to systematically screen public schoolchildren for unhealthy weight status. This study aims to examine the socioeconomic disparities in Body Mass Index (BMI) growth and the risk of the onset of obesity from childhood through adolescence.

Methods: This study analyzed (in 2015) the data for a large cohort of Arkansas public schoolchildren for whom BMIs were measured from school years 2003/2004 through 2009/2010. A linear growth curve model was used to assess how child-level sociodemographics and neighborhood characteristics were associated with growth in BMI z-scores. Cox regression was subsequently used to investigate how these factors were associated with the onset of obesity. Because children might be classified as obese in multiple years, sensitivity analysis was conducted using recurrent event Cox regression.

Results: Survival analysis indicated that the risk of onset of obesity rose sharply between ages of 5 and 10 and then again after age 15 . The socioeconomic disparities in obesity risk persisted from kindergarten through adolescence. While better access to full service restaurants was associated with lower risk of the onset of obesity (Hazard Ratio $(\mathrm{HR})=0.98,95 \% \mathrm{CI}=0.97$ 0.99), proximity to fast food restaurants was related to increased risk of the onset of obesity (HR $=1.01,95 \% \mathrm{CI}=1.00-1.01)$.
\end{abstract}

Conclusions: This analysis stresses the need for policies to narrow the socioeconomic gradient and identifies important time periods for preventative interventions in childhood obesity.

Key words: health disparities; BMI growth; onset of obesity; food environment 


\section{Introduction}

According to the 2011 National Survey of Children's Health (NSCH), about 20\% of children ages 10 to 17 in Arkansas were obese, which was almost 10 percentage points higher than the states with lowest childhood obesity rates (Levi et al., 2014). Longitudinal studies indicate that, overall, about half of obese school-age children subsequently become obese adults, although estimates differ across studies (Serdula et al., 1993). Understanding the determinants of childhood obesity in the state of Arkansas is thus critical to developing policies for early intervention and prevention of adult obesity in this state-specific population.

A multitude of recent studies have documented the socioeconomic gradient in childhood obesity in the United States, and some indicate that the gradient has increased since 2003 (Frederick et al., 2014; Singh et al., 2010). This could be related to the underlying socioeconomic disparities in food intake and physical activities. A recent review found that low-income children who participated in the Supplemental Nutrition Assistance Program (SNAP) consumed a poorer diet than higher-income non-participating children (Andreyeva et al., 2015). Another review of adolescent physical activity by socioeconomic status indicated that adolescents with higher SES had more physical activities than those with low SES, although discordant findings also existed (Stalsberg and Pedersen, 2010).

Another concern is that low-income children lack access to healthy foods. A review by Cobb et al. (2015) suggested that exposure to fast food around children's home was consistently found to be positively associated with obesity among low-income populations. This agrees with the conclusion from another review by Williams et al. (2014) who found evidence of a positive association between fast food availability surrounding children's schools and obesity. However, there are limitations to some of the studies included in the review, including a paucity of longitudinal studies, use of self-reported weight outcomes, and absence of individually specific exposure measures.

We employed a large longitudinal cohort in Arkansas based on objectively measured BMI z-scores and incorporated survival analysis into the study of obesity. This method has been used to study the role of naturalistic weight-reduction efforts in obesity prevention (Stice et al., 1999), but it has rarely been used in the examination of the roles of socioeconomic status and food environment in childhood obesity. A recent study based on data on public schoolchildren in 
Arkansas suggested that exposure to food desert was positively associated with BMI z-scores (Thomsen et al., 2016).

The aims of this study are to 1) examine BMI growth trajectories among a cohort of children; 2) assess the risk of the onset of obesity among this cohort over the study period; and 3) investigate the socioeconomic, gender, and racial disparities in BMI growth and the risk of the onset of obesity while controlling for food environment and other factors.

\section{Methods}

\section{Study Sample}

Due to concerns over the rise in childhood obesity, the Arkansas General Assembly passed Act 1220 of 2003, which required that schools conduct annual BMI screenings. From school years 2003/2004 through 2006/2007, all public school children from kindergarten through $12^{\text {th }}$ grade in the state of Arkansas were screened, but since school year 2007/2008, only children in even grades $(\mathrm{K}, 2,4,6,8,10)$ were screened. The height and weight measurements of schoolchildren were collected by trained personnel who followed statewide protocols to ensure uniformity in both measurement procedures and equipment. This study analyzed a longitudinal dataset on all children who were in kindergarten, second or fourth grades in academic year $2003 / 2004$ and who could be followed through subsequent waves of data collection $(\mathrm{N}=21,639)$ until academic year 2009/2010.

\section{Measures}

BMI was calculated as a ratio ([weight in pounds / (height in inches) $\left.)^{2}\right] \times 703$ ) and was then converted to age-gender specific z-scores following guidelines from the Centers for Disease Control and Prevention. Obesity was defined as a BMI greater than or equal to the 95th percentile for children of the same age and sex. The $95^{\text {th }}$ percentile corresponds to a BMI z-score of 1.645 .

The BMI dataset also contained basic sociodemographic information including age, gender, and race/ethnicity. School meal status was a dichotomous variable indicating whether the child qualified for free or reduced price lunches. School meal status has been widely used as a proxy for socioeconomic status. 
A measure of the child's food desert status indicated whether a student lived in a food desert. Our definition of a food desert was based on that used by the USDA Food Access Research Atlas except that we used a food desert measure that is specific to each child's residence.

The BMI data were geocoded and matched with food store locations by personnel from the Arkansas Center for Health Improvement (ACHI). The numbers of full service, and fast food restaurants (including fast food restaurants, and other limited service restaurants such as sandwich and pizza restaurants) were measured as counts along the most direct street route from the child's residence to the school within a 50 meter buffer on either side of the street. Further details on this measure are available elsewhere (Dunn et al., 2015).

Additional neighborhood characteristics were obtained from 2005-2009 American Community Survey (ACS) and were measured at the census block-group level. Specifically, we controlled for neighborhood educational attainment, proportion of working mothers, proportion of population below poverty level, housing vacancy rates, and proportion with access to vehicles.

\section{Statistical Analysis}

A growth curve model was used to examine the trajectories of children's BMI z-scores over the six BMI measurement occasions, and how child-level sociodemographics and neighborhood characteristics were associated with BMI z-score growth. Initial examination of these data suggested that the mean BMI z-scores followed a nearly linear growth path over time. Likelihood-ratio tests indicated that a growth curve model was preferred to ordinary least squares. To complement the analysis using the growth curve model, we also assessed the onset of obesity using Cox regression analysis while controlling for child-level sociodemographics and neighborhood characteristics. We first examined the time to the first occurrence of obesity using Cox regression. Because it is possible for a child to be classified as obese in some periods but not in others, we also examine obesity status as a recurrent event to test the sensitivity of the model. To investigate the socioeconomic disparities in the risk of the onset of obesity, the hazard of obesity was estimated by meal status based on the single event Cox regression. Observations with missing values in any of the variables were excluded from the analyses. All analyses were conducted using the Stata® software package, version 14. 


\section{Results}

The overall average BMI z-score was 0.68 across all years (Table 1) and increased almost linearly from 0.63 in academic year 2003/2004 to 0.74 in academic year 2009/2010.

Consequently, obesity prevalence also increased as our cohorts aged. The average across all cohorts increased from $17.96 \%$ (2003/2004) to $22.21 \%$ (2009/2010), with an average of $20.60 \%$ across the study period. 
Table 1 Descriptive statistics of variables and results from regression analyses

\begin{tabular}{|c|c|c|c|c|c|c|c|c|}
\hline \multirow[b]{2}{*}{ Variable name } & \multirow[b]{2}{*}{ Mean or $\%$} & \multirow[b]{2}{*}{ S.D. } & \multicolumn{2}{|l|}{ Growth curve model } & \multicolumn{2}{|l|}{ Cox regression } & \multicolumn{2}{|c|}{ Cox regression (recurrent event) } \\
\hline & & & Coefficient & $95 \% \mathrm{CI}$ & Hazard Ratio & $95 \% \mathrm{CI}$ & Hazard Ratio & $95 \% \mathrm{CI}$ \\
\hline BMI z-score & 0.68 & 1.06 & & & & & & \\
\hline Obese status & $20.60 \%$ & & & & & & & \\
\hline Free or reduced meal status & $48.00 \%$ & & 0.01 & $(0.00,0.02)^{* *}$ & 1.17 & $(1.10,1.24)^{* * *}$ & 1.09 & $(1.03,1.14)^{* * *}$ \\
\hline Female & $48.86 \%$ & & -0.11 & $(-0.13,-0.08)^{* * *}$ & 0.85 & $(0.81,0.90)^{* * *}$ & 0.92 & $(0.88,0.97)^{* *}$ \\
\hline White (reference) & $69.66 \%$ & & & & & & & \\
\hline Black & $22.53 \%$ & & 0.17 & $(0.14,0.20)^{* * *}$ & 1.21 & $(1.13,1.29)^{* * *}$ & 1.38 & $(1.29,1.48)^{* * *}$ \\
\hline Hispanic & $6.15 \%$ & & 0.20 & $(0.16,0.24)^{* * *}$ & 1.51 & $(1.37,1.66)^{* * * *}$ & 1.61 & $(1.47,1.76)^{* * *}$ \\
\hline Other race & $1.82 \%$ & & 0.08 & $(0.04,0.11)^{* * *}$ & 1.01 & $(0.83,1.24)$ & 1.12 & $(0.94,1.34)$ \\
\hline Food desert & $9.52 \%$ & & -0.01 & $(-0.02,0.01)$ & 1.01 & $(0.93,1.09)$ & 0.99 & $(0.92,1.06)$ \\
\hline Full service restaurants & 1.56 & 4.57 & -0.0005 & $(-0.0017,0.0007)$ & 0.98 & $(0.97,0.99)^{* *}$ & 0.99 & $(0.98,1.00)^{* *}$ \\
\hline Fast food restaurants & 1.45 & 10.48 & 0.0001 & $(-0.0004,0.0007)$ & 1.01 & $(1.00,1.01)^{* *}$ & 1.00 & $(1.00,1.01)^{*}$ \\
\hline High school graduate or higher & 0.36 & 0.11 & -0.01 & $(-0.08,0.05)$ & 0.88 & $(0.64,1.21)$ & 0.98 & $(0.73,1.30)$ \\
\hline Some college education & 0.27 & 0.09 & -0.02 & $(-0.08,0.05)$ & 0.83 & $(0.60,1.16)$ & 0.91 & $(0.68,1.23)$ \\
\hline College graduate or higher & 0.18 & 0.13 & -0.10 & $(-0.16,-0.04) * * *$ & 0.34 & $(0.25,0.45)^{* * *}$ & 0.38 & $(0.29,0.50)^{* * *}$ \\
\hline Working mother & 0.25 & 0.20 & 0.02 & $(0.00,0.05) \dagger$ & 1.11 & $(0.98,1.27)$ & 1.12 & $(1.00,1.26) \dagger$ \\
\hline Income below poverty & 0.18 & 0.14 & 0.05 & $(0.01,0.10)^{*}$ & 1.04 & $(0.82,1.32)$ & 1.02 & $(0.82,1.26)$ \\
\hline Vacant housing unit & 0.12 & 0.10 & -0.01 & $(-0.06,0.05)$ & 0.84 & $(0.65,1.09)$ & 0.84 & $(0.66,1.07)$ \\
\hline No vehicle & 0.07 & 0.08 & -0.01 & $(-0.08,0.06)$ & 1.24 & $(0.87,1.76)$ & 0.95 & $(0.70,1.30)$ \\
\hline Years since 2004 & & & 0.02 & $(0.02,0.02) * * * *$ & & & & \\
\hline Constant & & & 0.64 & $(0.58,0.69)^{* * *}$ & & & & \\
\hline
\end{tabular}


Results from the growth curve model indicated that the average BMI z-score for children in the free or reduced price meal group was about 0.01 higher than the comparison group. Girls, in general, had lower BMI z-scores than boys. Compared to whites, all the other racial/ethnic groups including blacks, Hispanics, and others had higher BMI z-scores, with Hispanics being the highest among all the groups. Living in neighborhoods featuring higher proportions of people with a college degree or higher was negatively associated with BMI z-scores; whereas living in neighborhoods with higher proportions of working mothers and people below poverty level were positively associated with BMI z-scores.

Cox regression indicated that the hazard for the onset of obesity over the study period for children in the free or reduced price meal group was about $17 \%$ higher than the full price meal group $($ Hazard Ratio $(H R)=1.17,95 \% \mathrm{CI}=1.10-1.24)$. This model-implied obesity hazard measures the instantaneous risk of obesity, given that obesity has not happened yet. It rose from age 5 to 10, decreased thereafter before rising sharply at about 15 years of age (Figure 1). Girls had lower hazard for the onset of obesity than boys. Blacks and Hispanics had higher hazard for the onset of obesity than whites during the study period. Better access to full service restaurants on the route from a student's home to school was associated with lower hazard for the onset of obesity over the study period, whereas fast food restaurant was associated with higher hazard. With exception of one educational attainment measure, other neighborhood level characteristics were not significantly associated with the onset of obesity. Treating obesity as a recurrent event in the Cox regression yielded similar results. 
Figure 1 Estimated obesity hazard by meal status. Analyses were based on data for a large cohort of Arkansas public schoolchildren for whom BMIs were measured from school years 2003/2004 through 2009/2010.

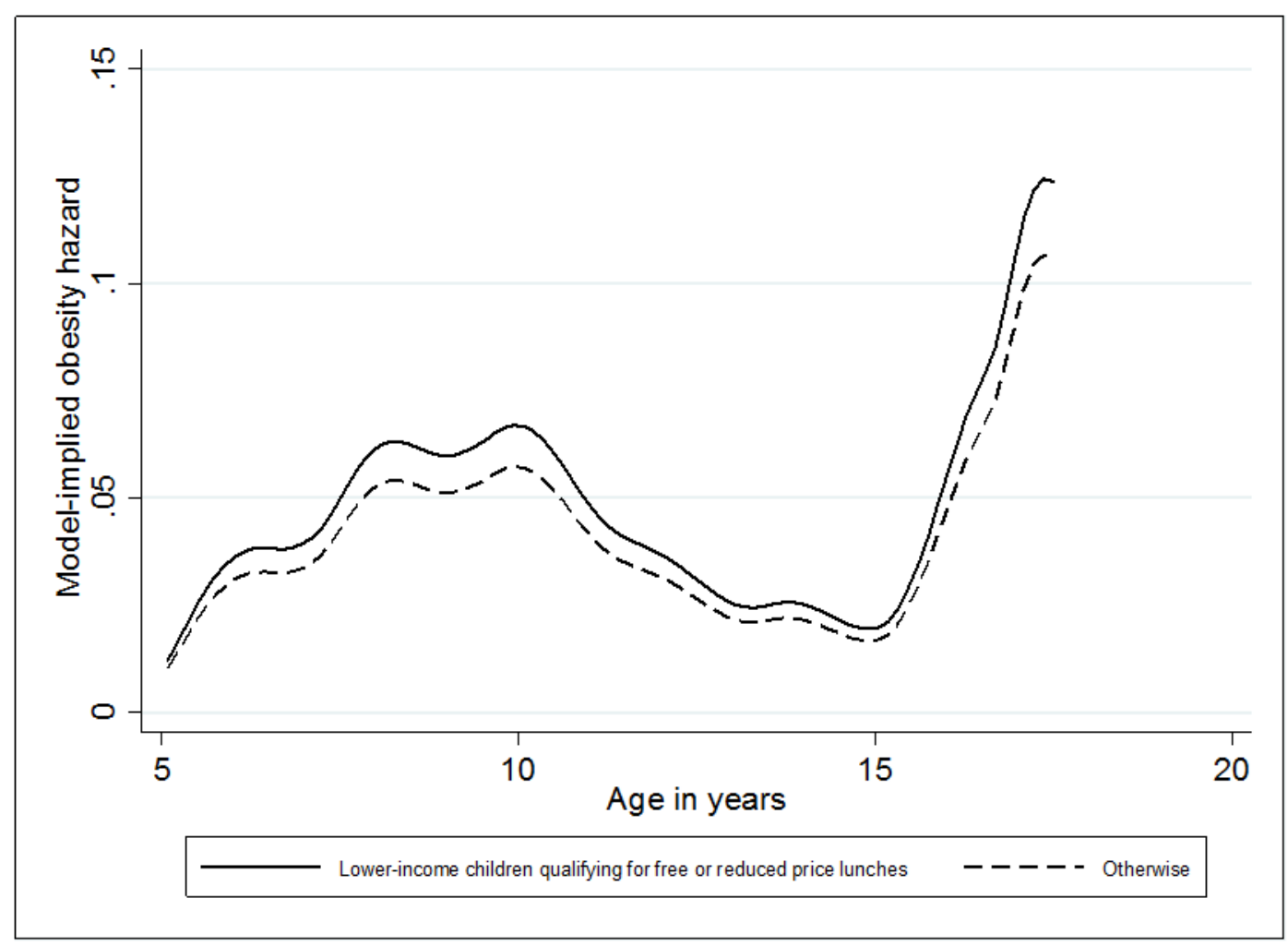




\section{Discussion}

This paper innovatively incorporated survival analysis into the study of obesity. Both the growth curve model and the cox regression indicated significant socioeconomic disparities in obesity risk. The cox regression also picked up significant association between access to restaurants and the onset of obesity, and yielded the changes in obesity hazard over age.

The finding that children who qualify for free or reduced price lunches at school had higher BMI growth and risk of obesity than those who pay full prices agrees with other studies based on different samples (Rundle et al., 2012). One possible explanation for this finding is that households living in low-income communities often have poorer access to healthy food options via supermarkets and grocery stores, but are exposed to more fast food restaurants in the neighborhood (Larson et al., 2009). Energy-dense foods are also often relatively cheaper than healthier alternatives such as fruits and vegetables (Monsivais and Drewnowski, 2009). In addition, children from low income families are more likely to reside in neighborhoods with safety issues and fewer amenities (e.g. playgrounds, sidewalks, recreational facilities) which prevent them from maintaining sufficient physical activities (Gordon-Larsen et al., 2006).

The gender and racial/ethnic differences in BMI growth and obesity risk among children in Arkansas are consistent with national observations (Ogden et al., 2014; Singh et al., 2010). Earlier studies found that compared to whites, black and Hispanic children were more likely to be exposed to maternal depression before birth, eat solid foods before 4 months of age, experience restrictive feeding practices during infancy, have television exposure in the bedroom, and have higher intake of sugar-sweetened beverages and fast food after 2 years of age (Taveras et al., 2010).

Better access to full service restaurants was negatively associated with the onset of obesity in our study. This concurred with evidence from Mellor et al. (2011) that proximity to full service restaurants within 0.1 mile radius of children's residence was associated with decreased odds of obesity. The positive association between availability of fast food restaurants and onset of obesity agreed with past reviews (Cobb et al., 2015; Williams et al., 2014).

We found that the risk of the onset of obesity increased from around ages 5 to 10 , decreased moderately between ages 10 and 15 and rose sharply after age 15 . A systematic review 

reason could be the changes in the food and physical environment that a school brings (Novotny et al., 2015). Based on the 2003-2004 NHANES, one study found that the level of physical activities among youth declined dramatically as age increased, independent of confounding factors such as gender, race/ethnicity, and socioeconomic status (Whitt-Glover et al., 2009).

This study has several limitations. First, the dataset did not contain important behavioral risk factors for obesity such as food intake and physical activity. Second, this study focused specifically on Arkansas children and so the results of this study may not be generalizable to the broader population of children in the United States. Third, BMI screenings were not conducted in academic year 2008/2009 for the cohorts of children followed through the study because of a statewide change to the frequency of the screening program. Fourth, BMI does not distinguish fat from nonfat mass such as bone and muscle, and is thus not a perfect measure of obesity. Due to data limitations, the potential impact of the use of a different measure of obesity on results cannot be assessed in this study. Although there were limitations in applying listwise deletion to data analysis, the loss of generalizability and bias in the estimates of our study was not serious for a couple of reasons. First, out of the 21,639 subjects being followed, less than $1 \%$ of them had missing values in one or more of the variables included in the model, which was low considering the large sample size. Second, the extent to which complete case analysis causes problems depends on the patterns of missing data. There was no clear evidence suggesting serious violation of the Missing Completely at Random (MCAR) assumption, as the BMI data were collected by trained personnel rather than self-reported.

\section{Conclusion}

The persistent socioeconomic disparities in BMI growth and obesity risk among public school children in Arkansas underscore the urgent need for school and community based interventions to narrow the gap, especially during high-risk periods. In addition, the socioeconomic disparities in exposure to obesogenic environment place children from disadvantaged families in higher risk for obesity and so it is important to implement policies aimed at improving the food and physical environment in low SES and minority neighborhoods. 


\section{Acknowledgements}

This work was funded by the Agriculture and Food Research Initiative Competitive Grant No. 2011-68001-30014 from the USDA National Institute of Food and Agriculture. This work is also partly supported by the Arkansas Biosciences Institute, Research Council of Norway Grant \# 233800, and the National Research Foundation of Korea (NRF-2014S1A3A2044459).

\section{References}

Andreyeva, T., Tripp, A.S., Schwartz, M.B., 2015. Dietary Quality of Americans by Supplemental Nutrition Assistance Program Participation Status: A Systematic Review. American journal of preventive medicine. Cobb, L.K., Appel, L.J., Franco, M., Jones - Smith, J.C., Nur, A., Anderson, C.A., 2015. The relationship of the local food environment with obesity: A systematic review of methods, study quality, and results. Obesity 23:1331-44.

Dunn, R., Thomsen, M., Nayga, J.R., Rouse, H., 2015. A longitudinal analysis of fast-food exposure on child weight outcomes: identifying causality through school transitions, Working Paper, Dept of Ag. \& Resource Econ, Univ. of Connecticut.

Frederick, C.B., Snellman, K., Putnam, R.D., 2014. Increasing socioeconomic disparities in adolescent obesity. Proceedings of the National Academy of Sciences 111:1338-42.

Gordon-Larsen, P., Nelson, M.C., Page, P., Popkin, B.M., 2006. Inequality in the built environment underlies key health disparities in physical activity and obesity. Pediatrics 117:417-24.

Larson, N.I., Story, M.T., Nelson, M.C., 2009. Neighborhood environments: disparities in access to healthy foods in the US. American journal of preventive medicine 36:74-81. e10.

Levi, J., Segal, L., St Laurent, R., Rayburn, J., 2014. The state of obesity: better policies for a healthier America. Washington, DC: Trust for America's Health.

Mellor, J.M., Dolan, C.B., Rapoport, R.B., 2011. Child body mass index, obesity, and proximity to fast food restaurants. International Journal of Pediatric Obesity 6:60-68.

Monsivais, P., Drewnowski, A., 2009. Lower-energy-density diets are associated with higher monetary costs per kilocalorie and are consumed by women of higher socioeconomic status. Journal of the American Dietetic Association 109:814-22.

Novotny, R., Fialkowski, M.K., Li, F., Paulino, Y., Vargo, D., Jim, R., Coleman, P., Bersamin, A., Nigg, C.R., et al., 2015. Systematic Review of Prevalence of Young Child Overweight and Obesity in the United States-Affiliated Pacific Region Compared With the 48 Contiguous States: The Children's Healthy Living Program. American journal of public health 105:e22-e35.

Ogden, C.L., Carroll, M.D., Kit, B.K., Flegal, K.M., 2014. Prevalence of childhood and adult obesity in the United States, 2011-2012. Jama 311:806-14.

Rundle, A., Richards, C., Bader, M.D., Schwartz-Soicher, O., Lee, K.K., Quinn, J., Lovasi, G.S., Weiss, C., Neckerman, K., 2012. Individual-and school-level sociodemographic predictors of obesity among New York City public school children. American journal of epidemiology 176:986-94. 
Serdula, M.K., Ivery, D., Coates, R.J., Freedman, D.S., Williamson, D.F., Byers, T., 1993. Do obese children become obese adults? A review of the literature. Preventive medicine 22:167-77.

Singh, G.K., Siahpush, M., Kogan, M.D., 2010. Rising social inequalities in US childhood obesity, 20032007. Annals of epidemiology 20:40-52.

Stalsberg, R., Pedersen, A.V., 2010. Effects of socioeconomic status on the physical activity in adolescents: a systematic review of the evidence. Scandinavian journal of medicine \& science in sports 20:368-83.

Stice, E., Cameron, R.P., Killen, J.D., Hayward, C., Taylor, C.B., 1999. Naturalistic weight-reduction efforts prospectively predict growth in relative weight and onset of obesity among female adolescents. Journal of consulting and clinical psychology 67:967.

Taveras, E.M., Gillman, M.W., Kleinman, K., Rich-Edwards, J.W., Rifas-Shiman, S.L., 2010. Racial/ethnic differences in early-life risk factors for childhood obesity. Pediatrics 125:686-95.

Thomsen, M.R., Nayga, R.M., Alviola, P.A., Rouse, H.L., 2016. The Effect of Food Deserts on the Body Mass Index of Elementary Schoolchildren. American Journal of Agricultural Economics 98:1-16.

Whitt-Glover, M.C., Taylor, W.C., Floyd, M.F., Yore, M.M., Yancey, A.K., Matthews, C.E., 2009. Disparities in physical activity and sedentary behaviors among US children and adolescents: prevalence, correlates, and intervention implications. Journal of Public Health Policy:S309-S34.

Williams, J., Scarborough, P., Matthews, A., Cowburn, G., Foster, C., Roberts, N., Rayner, M., 2014. A systematic review of the influence of the retail food environment around schools on obesity - related outcomes. obesity reviews 15:359-74. 


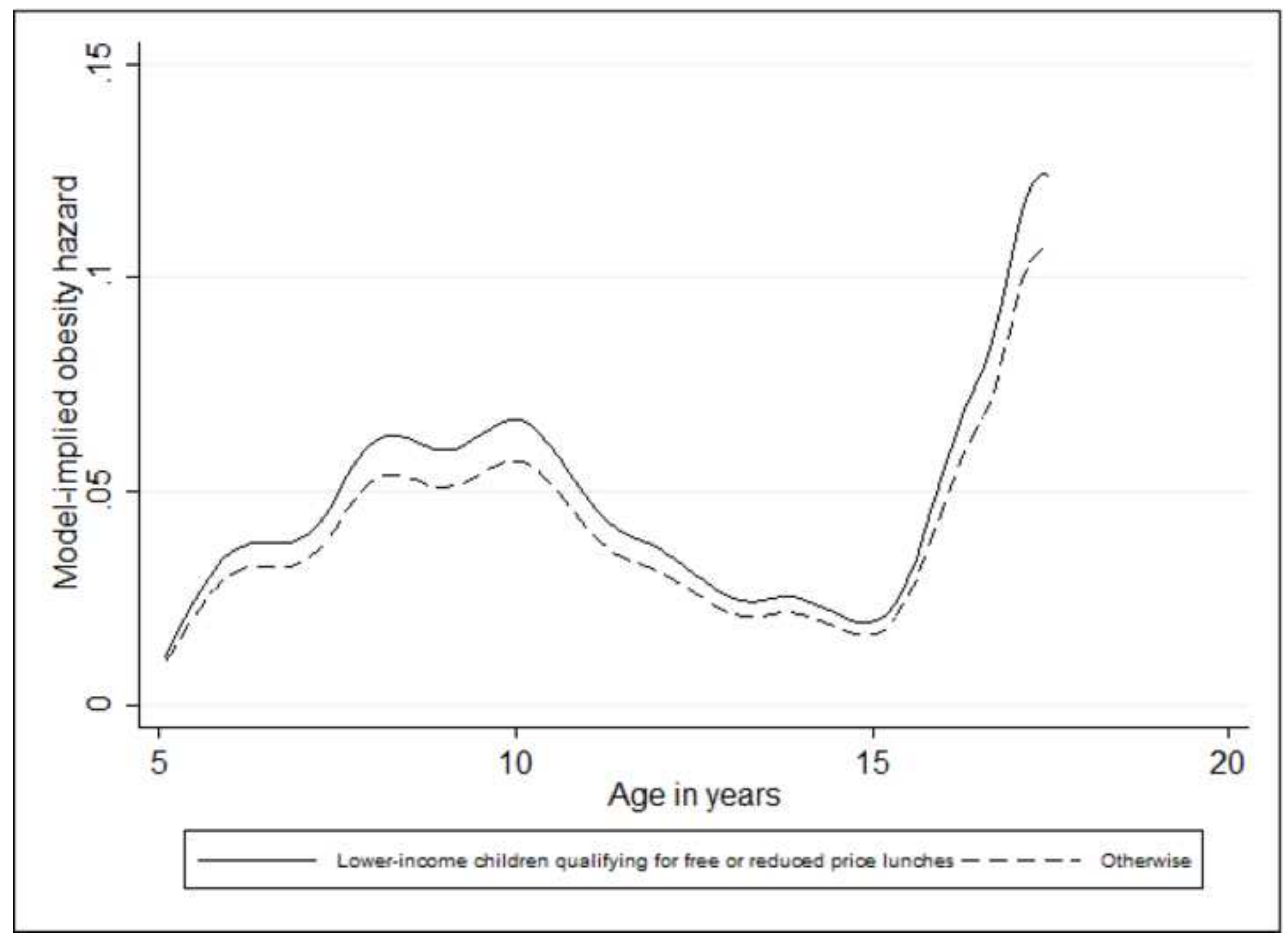

